Voix et Images

voixetimages

\title{
À la première personne qui m'écoutera
}

\section{DANIEL GRENIER}

Volume 43, numéro 1 (127), automne 2017

URI : https://id.erudit.org/iderudit/1043155ar

DOI : https://doi.org/10.7202/1043155ar

Aller au sommaire du numéro

\section{Éditeur(s)}

Université du Québec à Montréal

\section{ISSN}

0318-9201 (imprimé)

1705-933X (numérique)

Découvrir la revue

Citer ce compte rendu

GRENIER, D. (2017). Compte rendu de [À la première personne qui m'écoutera]. Voix et Images, 43(1), 119-125. https://doi.org/10.7202/1043155ar d'utilisation que vous pouvez consulter en ligne.

https://apropos.erudit.org/fr/usagers/politique-dutilisation/ 


\section{R O M A N}

À la première personne qui mécoutera

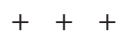

DANIEL GRENIER

Université Laval

Fanie Demeule, Kevin Lambert, Antoine Charbonneau-Demers. La première est née en 1990, le deuxième en 1992, le troisième en 1994. Ils publient tous trois leur premier roman, respectivement chez Hamac, chez Héliotrope et chez VLB. Des romans courts, à l'imagerie incisive et aux propos cyniques, que les critiques ont qualifiés de "coup[s] de poing», et dont les auteurs ont été vus, qui comme une «surprise», qui comme une sorte de "prophète de la destruction".

Le fameux "coup de poing", on sait ce qu'il veut dire. Il est utilisé la plupart du temps pour décrire des récits de fiction ou d'autofiction assez courts, qui (pour utiliser un autre cliché à la peau dure) ne nous laissent ni indemnes ni indifférents. Le texte, ici, est considéré comme presque dangereux, au sens où il nous «frappe de plein fouet» en nous révélant des choses (sur nous, sur notre société) qui étaient jusque-là restées taboues. Nous ne serons plus les mêmes après la lecture: le livre nous aura changés. Et il nous aura donné l'envie de changer le monde. Ce n'est pas tant la prose qui claque (elle peut même être assez classique) que le sujet qui agit comme un électrochoc. Demeule, dans Déterrer les $o s^{1}, s^{\prime}$ intéresse aux troubles alimentaires qui affectent la vie d'une jeune femme et vient interroger notre rapport maladif à la nourriture. Ce qui s'ouvre comme la confession d'un individu en lutte avec des démons bien personnels se transforme rapidement en une charge acerbe contre la façon que nous avons tous et toutes de consommer et de jeter.

L'impression de "surprise», voire de "révélation», émerge de l'idée qu'on se fait souvent des jeunes écrivains et écrivaines qui publient pour la première fois: ils sortent de nulle part, tout en arrivant à point nommé, et cette sortie est éclatante, aveuglante, parce qu'on ne l'avait pas prévue. Personne ne nous avait averti qu'un tel talent précoce se dissimulait dans les combles de l'édition québécoise. Antoine Charbonneau-Demers, avec ses vingt-trois ans et son prix Robert-Cliche du premier roman pour $\mathrm{CoCo}^{2}$, fait ici figure d'exemple, lui qui précise en entrevue avoir

1 Fanie Demeule, Déterrer les os, Québec, Septentrion, coll. «Hamac», 2016, 112 p.

2 Antoine Charbonneau-Demers, Coco, Montréal, VLB éditeur, 2016, 215 p. 
le «syndrome de l'imposteur», puisque son ambition principale est d'être acteur de théâtre ${ }^{3}$. L'écriture romanesque devient par le fait même une sorte de seconde peau que l'on enfile avec un naturel qui fait son effet, qui semble couler de source.

De son côté, Kevin Lambert a asséné plus d'un coup de poing, d'après ce que je lis dans les journaux, avec Tu aimeras ce que tu as tuét. De nombreux critiques se sont dits sonnés par la prose cinglante du jeune romancier, ainsi que par les idées véhiculées dans l'œuvre, laquelle aurait ni plus ni moins la force de frappe d'une salve de mitraillette. Le ton «apocalyptique» se retrouve chez Lambert dès le titre, en effet, qui annonce, à la fois par son adresse à un interlocuteur («Tu») et par l'usage du futur simple, un avènement dont la complexité reste à voir, un carnavalesque revirement des valeurs: la mort et l'amour, intervertis, chamboulés.

Ces trois-là partagent bien sûr davantage que les lieux communs malheureux de la critique impressionniste. Si je les ai réunis ici, c'est pour examiner un des aspects de leurs livres, soit leur narration autodiégétique au présent de l'indicatif. En critique romanesque, la voix narrative n'est peut-être pas l'élément qui soulève le plus de passion, elle perd souvent au change contre l'intérêt porté au récit et à la force des images; mais pour moi, rien n'apparaît plus important et stimulant que de se pencher sur cette dimension particulière de l'œuvre. Il s'agit d'un choix opéré par l'auteure ou l'auteur, une décision délibérée et consciente, qui permet sans aucun doute, sinon de comprendre sa portée, au moins d'analyser le projet d'un livre donné, la direction qu'il a prise et sa destination ultime.

Chaque fois que je rencontre ce type de narration, je suis étonné et fasciné par ce «je» qui s'exprime au présent. Demeule, Lambert et Charbonneau-Demers ne sont pas les premiers à s'en servir, bien au contraire. Ils participent d'une esthétique prisée par plusieurs en littérature québécoise, celle de l'intimité et de la proximité entre narrateur et narrataire que permet la première personne. Dans ma chronique précédente $^{5}$, je mentionnais ce même type de narration dans le cas du roman de Christian Guay-Poliquin, Le poids de la neige (La Peuplade, 2016), et je n'ai de cesse de le croiser chez les nouvelles plumes faisant leur entrée dans le milieu littéraire. Chez Chloé Savoie-Bernard, Nicholas Giguère, Katherine Raymond, Steph Rivard, etc. C'est le caractère profondément indécidable de cette voix narrative qui m'intéresse ici, sa nature flottante qui oscille entre la pensée qui se construit en direct et l'écrit rétrospectif et analytique.

Observons les incipits.

Chez Demeule: «Je t’ai déjà dit que je suis arrivée légèrement d'avance. Je pèse quatre livres. Je bronze sous l'incubateur pendant un bon moment, intubée comme un petit poulet qu'il faut vite engraisser. Une fois sortie de l'hôpital, je ne reste pas

3 Voir Marie-France Bornais, «Une candidature de dernière minute qui s'avère payante», Journal de Québec, 9 octobre 2016, en ligne: http://www.journaldequebec.com/2016/10/09/une-candidature-de-derniereminute-qui-savere-payante (page consultée le 10 octobre 2017).

4 Kevin Lambert, Tu aimeras ce que tu as tué, Montréal, Héliotrope, 2017, 209 p.

5 Daniel Grenier, «Sous la neige et les bombes», Voix et Images, vol. XLII, n 2, hiver 2017, p. 161-167. 
chicot longtemps. Je tète sans arrêt.» (9) La temporalité de ce passage est paradoxale, puisque ce qui commence par une adresse à un «tu» qui évoque un temps passé, en l'occurrence celui de la naissance, est aussitôt suivi par une description en direct des événements vécus durant cette naissance. La narratrice, ici, pense des choses tout en les évoquant simultanément. Autrement dit, elle est ce bébé qui dit un peu plus loin: « Je suis un bébé en mode aspirateur» (9), tout en étant la personne adulte qui regarde ce bébé en retranscrivant ce qu'il pourrait avoir pensé. Ce double état sera reconduit tout au long du livre, et l'effet de distance s'amenuisera à mesure que le temps du récit et le temps de l'écriture se rapprocheront. Demeule, en optant pour ce temps grammatical, construit sa narration sur l'idée du souvenir qui hante, qui prend plus de place que la réalité présente, jusqu'à envahir à la fois l'esprit et la mémoire.

Ensuite, chez Charbonneau-Demers: «Mon père stationne la voiture devant l'ancien presbytère, où deux ombres discutent à la fenêtre derrière un rideau de dentelle jaune. Il avait refusé de me dire où il m'emmenait, et il s'était mis à pleuvoir sur le chemin. Il a la peau du visage trouée, mon père, criblée de remords et d'attente.» (9) Encore une fois, c'est à une double temporalité que l'on a affaire ici, alors que ce père et ce fils se font face dans la voiture et dans l'écriture. Au moment décrit, le narrateur, qui sera plus tard surnommé Coco, a une dizaine d'années, mais ses observations (sur le visage de son père, sur ses joues creusées par les remords) sont celles d'une entité nécessairement plus mature, aux propos plus acérés, en contrôle de ses métaphores, qu'on ne rencontrera jamais vraiment à l'intérieur de l'économie du livre. Cette entité sera toujours présente, pourtant, dans les propos tenus par le narrateur-enfant, dont la voix sera marquée d'un côté par une naïveté reproduisant l'immaturité et de l'autre par un sens de l'ironie et une causticité attribuables au détachement du souvenir.

Finalement, chez Lambert: «Dans la classe, la professeure entre. Elle porte une canne et son autorité fêlée, on rit d'elle dans son dos, la vieille sorcière, on est en deuxième année.» (11) Par l'inversion syntaxique assez osée et le zeugme (autorité; canne) qui la suit immédiatement, on constate illico que le narrateur, ici, est à la fois très proche et très loin de ce qu'il raconte. On a affaire en même temps à un gamin ayant du mal à contrôler ses figures de style, qui se moque de sa professeure avec ses camarades de classe, et à un jeune écrivain en pleine possession de ses moyens, qui revient sur des événements du passé. Impossible de savoir lequel des deux est réellement aux commandes de la narration, puisque Lambert s'amuse à brouiller les pistes constamment: ce que nous lisons est-il écrit, composé a posteriori, où est-il simplement vécu? Contrairement à ses collègues Demeule et Charbonneau-Demers, toutefois, Lambert sème ici et là des traces assez explicites d'un temps de l'écriture postérieur. Son narrateur nommé Faldistoire y va de phrases énigmatiques comme: «Plus loin dans l'histoire, je serai confronté tous les jours à ses folies fantomales quand ma mère mourra et que je serai forcé d'aller vivre chez mes grands-parents, à Rivière-du-Moulin, jusqu'à mes douze ans.» (32) Rapidement, d'ailleurs, la temporalité du récit de Faldistoire se fait délibérément brumeuse, alors que différents moments sont racontés simultanément.

Ces quelques particularités narratives apportent avec elles ce qu'il convient d'appeler un mode commun: celui de l'onirisme. Cette première personne du 
singulier qui s'exprime, en effet, c'est celle qu'on utilise quand on décrit un rêve, et les récits qui m'occupent ici ont tendance à passer du réalisme le plus cru au surréalisme assumé, sans avertissement. C'est-à-dire qu'ils portent en eux la promesse d'un univers diégétique auquel les lecteurs adhèrent au départ, mais qui pourrait se déglinguer d'un moment à l'autre pour sombrer dans le rêve ou le cauchemar. De la même manière que la temporalité du récit et celle de l'écriture tendent à fusionner, le réel et le fantasme s'imbriquent jusqu'à devenir inextricables. Une scène de Déterrer les os, de Demeule, est particulièrement éloquente à cet égard, alors que la mort s'immisce soudain dans le récit de vie de cette jeune femme qui a pris la parole pour nous décrire son angoisse existentielle. De vivante, elle passe à morte et devient un corps, tout en conservant la faculté de ressentir et de dire:

La journée a été chaude et le temps est pesant. Ça sent les herbes fraîchement fauchées. J'ai les écouteurs enfoncés dans les oreilles. Ma mère apparaît dans le cadre de la porte patio. Le souper est prêt. Je ne bouge pas. Ma mère s'impatiente, me crie dessus plus fort. Je reste immobile, une revue ouverte sur les genoux. Ma mère sort, elle s'avance vers moi en maugréant contre les maudits écouteurs parce qu'il faut toujours s'époumoner à cause d'eux.

C'est là qu'elle se rend compte que quelque chose cloche. Elle s'arrête. Observe l'angle erratique de mon cou, ma tête tombée sur le côté, détachée. Sa main s'empare de mon épaule pour la secouer. Mon corps s'affale, révélant mon visage aux orbites blanches et à la bouche béante.

Ma mère recule d'un bond en hurlant.

Mon père intervient, me brasse comme on secouerait un pommier et mon crâne revole dans tous les sens. Un peu de bave gicle sur ma joue. Mon père répète «Non, non, non, non.» Mon frère et ma sœur se lèvent de table, mais restent hébétés derrière le portique entrouvert. Ma mère gît au sol.

Une bruine légère se met à tomber. Mon père renonce. C'est fini. Les pages de ma revue Seventeen tournent sous les bourrasques de vent, zootrope de nymphettes sveltes et souriantes.

On étend mon corps dans le gazon ras et piquant, tapissé de pissenlits chiffonnés. Mon père m'enroule dans la toile de protection de la piscine hors terre. Il laisse seulement émerger mon visage au bout, parce que c'est la dernière chose que l'on veut voir disparaître chez quelqu'un. On pleure autour de mon suaire, sauf ma mère qui demeure évanouie.

À trois, ils hissent mon corps dans le sapin derrière chez nous, sur ses hautes branches qui donnent en plongée sur la fenêtre de la cuisine. Lorsque ma mère fera la vaisselle ce soir, ce sont mes yeux qui l'éclaireront. Mon visage s'émaciera de plus en plus, jusqu'à s'effacer définitivement. (108-109)

On pourrait presque entendre la voix qui ajoute au début de la scène: «J'ai fait un rêve bizarre... je te le raconte. » Et pourtant, non. Ici, comme chez Lambert et chez Demers-Charbonneau, c'est dans le non-dit que se jouent la disparition soudaine des repères et la dissolution d'une identité. Cette façon de faire se rencontrer la réalité et le fantasme, de les mettre sur un même plan, de les faire se croiser jusqu'à 
assumer une seule et même forme, un seul et même ton, sert à illustrer l'arbitraire du monde, voire son absurdité. Demeule et ses collègues assument volontiers ce nivellement: en-dehors de celui-ci, point de salut, point de littérature. La vie d'un individu ne saurait se résumer aux événements réels qui la ponctuent, il faut également en montrer les illusions, les fantasmes et les fantômes. De là le ver solitaire qui dévore tranquillement mais sûrement les entrailles du narrateur de Coco; de là les enfants revenants qui peuplent la ville de Chicoutimi, dans Tu aimeras ce que tu as tué, et qui y reviennent pour mieux la faire exploser.

Paradoxalement, ces livres qui carburent à l'onirique et à l'incantatoire puisent leur force d'évocation dans la multiplication des effets de réel. L'usage d'anthroponymes, par exemple, est très important, dans la mesure où il vient contrebalancer l'anonymat ou l'absence de personnalité tangible des narrateurs, ou encore le flou entourant les lieux du récit. Lorsque, dans Coco, les personnages se mettent à imiter les prouesses physiques d'Anne-Marie Losique (58), c'est la communauté télévisuelle québécoise qui est sollicitée et qui nous offre un point d'appui où poser l'indécidabilité de cette petite ville éloignée des grands centres, là où se situe l'intrigue - cette ville, jamais nommée, désignée simplement par le syntagme péjoratif «racoin du monde» (17). À l'inverse, dans Tu aimeras ce que tu as tué, c'est le "racoin » lui-même qui fait office d'effet de réel catalyseur: la ville de Chicoutimi, plus que sommairement nommée, devient un personnage de l'histoire, une entité tentaculaire, créant une disparité non seulement avec la description des événements aussi terrifiants qu'abracadabrants qui y ont lieu, mais également avec l'existence réelle de la ville dans la topographie québécoise. Sans parler du fait que le narrateur choisit délibérément de semer la «destruction» (210), comme il le dit lui-même, à Chicoutimi, un espace géographique abondamment investi ces dernières années par des écrivains québécois ayant connu le succès, comme Geneviève Pettersen, Samuel Archibald et Larry Tremblay. Sous la plume de ces derniers, la ville du Saguenay n'était pas carrément glorifiée, mais on en exploitait le caractère quasi mythique, sa force d'évocation dans l'imaginaire social du Québec. Il va sans dire que c'est dans un tout autre registre que Kevin Lambert assène ses "coups de poing", en forme de scènes brèves, souvent violentes, qui se lisent comme des tentatives virulentes de révéler une vérité enfouie non pas dans notre imaginaire collectif, mais plutôt dans notre inconscient collectif : nous vivons dans une société qui repose sur des apparences et des faussetés. Sous ce vernis craquelant se cache notre véritable nature homophobe, raciste et toujours prête à écraser le plus faible. Lambert a également recours, comme Charbonneau-Demers, à des références culturelles communes, souvent choisies pour leur aspect populaire, voire "cucul ou kitsch», nous permettant par le fait même de nous approprier le discours lapidaire du narrateur tout en créant une distance salutaire entre les critiques de celui-ci et notre propre rapport au monde:

Toutes les fois qu'elle est nommée, elle lève son grand sourire plein de gencives pour aller montrer son nouveau linge et son beau t-shirt Mixmania devant tout le monde, et c'est d'une insupportable injustice de la voir remporter tous les honneurs, alors que plein d'élèves normaux auraient pu aussi gagner un certificat et les billets de cinéma donnés avec. (94) 
Oscillant constamment entre le mépris puéril de l'enfant (proche de l'intimidateur) et le rire sardonique de l'écrivain (proche du juge), le narrateur s'en tire à bon compte: sa misanthropie est justifiée, on la lui a injectée de force depuis sa naissance.

\section{$+$}

Stylistiquement parlant, les trois œuvres sont similaires, mais n'ont pas toutes le même impact. Par l'entremise de leur narration, toutes trois partagent un désir de créer à la lecture un effet d'instantanéité et de proximité. Le mode onirique y est privilégié dans la mesure où celui-ci procure une profondeur aux événements mondains qui font et défont une vie humaine. J'aimerais insister, en terminant, sur certaines caractéristiques qui distinguent les démarches respectives de ces jeunes écrivains. C'est à travers ces distinctions que se révèlent les forces de chacun de ces romans.

Les dialogues de Charbonneau-Demers, par exemple, sont extrêmement efficaces, rendus dans une oralité qui fait honneur à l'oreille du jeune écrivain. Non seulement sont-ils efficaces d'un point de vue esthétique, mais ils sont aussi extrêmement importants dans le rythme du récit, agissant presque comme de la ponctuation. Intercalées entre des blocs narratifs où l'écriture se fait plus conventionnelle, les lignes de dialogue sans incises dans Coco jouent d'abord sur les contrastes et les différents registres, ne souffrant ni du maniérisme qu'on retrouve souvent dans les romans québécois, ni de la grandiloquence souvent associée aux dialogues de thêâtre. Bien sûr, pour revenir à mes lubies narratologiques, on finit par se demander si ces paroles sont simplement prononcées dans le présent du récit ou si elles sont écrites dans le futur de l'écriture, mais ça n'enlève rien, au bout du compte, à leur exactitude. Voici un exemple, parmi tant d'autres, où on arrive à ressentir d'une manière très directe la complexité des sentiments de la mère du narrateur:

- Tu vas pas le faire?

- Pourquoi pas? Si j'ai besoin...

- Tu vas le faire?

- Je sais pas, oui...

- Est pas barrée de te demander ça... Je peux pas croire... Ça fait des mois que je te dis que ça serait plus beau court, pis tu m'écoutes pas, pis là une bonne femme inconnue t'a convaincu...

- Mais j'ai pas vraiment le choix.

- Oui, t'as toujours le choix mon amour.

- Ben ça me tente.

- Crisse... Tu vas te faire marcher sur les pieds toute ta vie, mon grand.

- Toi aussi tu veux que je me les fasse couper!

- OK, fais ce que tu veux... Mais on la connaît pas, cette coiffeuse-là, elle est peutêtre vraiment mauvaise.

- Maman...

- Quoi «maman»? Y'a pas de «maman», tu fais ce que tu veux, je t'ai dit.

- T'es fâchée.

- Non, c'est correct, je t'ai dit. (25) 
Du côté de Demeule, c'est la fluctuation entre les marqueurs de la culture d'élite et de la culture populaire qui étonnent et détonnent. Avec l'assurance postmoderne d'une écrivaine qui ne boude pas son plaisir bien qu'elle relate des événements lourds de conséquences, Demeule n'hésite pas à superposer les nombreuses influences (littéraires ou autres) qui sous-tendent son écriture, en multipliant les références et les hommages. Ici, c'est Hubert Aquin qui est subtilement cité, alors que la narratrice «retourne au fond des choses» (37); là, c'est la vie parallèle créée par le jeu vidéo Les Sims qui provoque la découverte par la narratrice de Marguerite Duras et des vies parallèles que son œuvre nous offre: "C'est plus décourageant qu'autre chose, à vrai dire, jouer aux Sims. [...] Je lis Moderato Cantabile sur la chaise longue dans ma cour au temps des déflagrations de magnolia. C'est mon premier Duras. Je crois tout saisir. Je deviens Anne Desbaresdes.» (45)

Des trois auteurs, Lambert est celui qui travaille le plus efficacement cette idée de rupture de ton qui, finalement, semble être un autre point commun aux trois œuvres. Dans Tu aimeras ce que tu as tué, le langage est toujours double et duel, il tire d'un côté et s'élance de l'autre, montrant les crocs. Les harangues emphatiques se disloquent pour laisser place à un babillage adolescent qui cède aussitôt le pas à une charge apocalyptique, la plupart du temps dans une même tirade:

J'étais attiré par sa peau foncée comme une insolence envers le racisme latent de Chicoutimi, ses cheveux et ses yeux noirs: un majeur long et raide enfoncé profond dans l'anus de notre charmante ville et remué jusqu'à sa jouissance abondante et involontaire. J'aimais l'effet esthétique de nos noms accolés: Almanach et Faldistoire, deux noms pas possibles [...]. Ses parents sont des craquepottes qui étudient les roches à l'université, qui les cognent avec leurs marteaux et les regardent avec leurs loupes, qui cherchent de l'or et des diamants, son père est même venu du Maroc juste pour cogner les roches de Chicoutte, il y en a des vraiment spéciales, ça a l'air, mais ça fait du chemin pareil pour regarder de la garnotte dans un microscope.» (102-103)

Il n'y a pas de résolution possible entre ces deux registres, mais c'est sans anicroche qu'ils cohabitent dans l'univers restreint de la fiction. Et c'est quelque part entre le «racisme latent de Chicoutimi», «l'anus de notre charmante ville» et «les roches de Chicoutte», quelque part entre la pure provocation et le cri primal, que se cache la vérité de ce narrateur fuyant et instable qu'est Faldistoire. Son créateur ne nous y donne jamais accès et nous abandonne à notre sort, entre ses mains. 